EPJ Web of Conferences 61, 06001 (2013)

DOI: $10.1051 /$ epjconf/ 20136106001

(C) Owned by the authors, published by EDP Sciences, 2013

\title{
Magnetic fields and polarization in AGN jets
}

\author{
John F. C. Wardle $e^{1, a}$ \\ ${ }^{1}$ Physics Department, Brandeis University, Waltham, Massachusetts 02454, U.S.A.
}

\begin{abstract}
In the literature there is now a wealth of images of AGN in both linear and circular polarization at milliarcsecond resolution of many sources at multiple epochs and wavelengths. This review is a broad overview of our current state of knowledge of the polarization properties of AGN jets, and of what we can infer about the structure of their magnetic fields and the distribution of Faraday rotating material, and also what is premature to infer. We suggest that while many of the observations of transverse rotation measure gradients may be correct, they have in general been observed with neither enough resolution nor sensitivity to be incontrovertible. Fortunately, this could be remedied using the new broad-band receivers on the VLBA. We also argue that while a transverse rotation measure gradient is a strong indicator of a toroidal component of magnetic field, it does not necessarily imply a helical magnetic field. In jets where the dominant field component appears to be parallel to the jet, it more likely consists of sheared loops or compressed random fields that are not vector ordered. Finally, we draw attention to the power of imaging in all four Stokes parameters at multiple frequencies to constrain the three-dimensional magnetic field structure and the particle content of jets and measure fundamental physical properties.
\end{abstract}

\section{Introduction}

The linear polarization of the radio emission from AGN jets (assumed to be synchrotron radiation) tells us about the structure and order of the magnetic field. Especially on parsec scales, these are intimately related to the launching, acceleration and collimation of the jets (see the review by D. Meier, these proceedings). Monitoring reveals the evolution of the magnetic field, and the formation and dissipation of shocks. Faraday rotation measurements are an important diagnostic of thermal electrons (and lower energy relativistic electrons) inside and outside the jet, and have revealed a torroidal component of the magnetic field in some sources. Observations of circular polarization can tell us about the positron fraction in the relativistic electron population and the vector-ordering of the magnetic field. The latter then permits an estimate of the net magnetic flux in the jet, and hence (because it is a conserved quantity) the magnetic flux at the central black hole-accretion disk system. It should be noted that none of the physical parameters listed in this paragraph can be gleaned from total intensity images.

With the advent of the VLBA in 1993, polarimetry at milliarcsecond resolution came of age. Earlier polarization results, and some of the first polarization results from the VLBA, were discussed in the 1997 Annual Reviews article by Zensus [1]. A conference celebrating the 10th birthday of the VLBA [2] featured 18 papers devoted to the polarization properties of AGN on parsec scales. These included observations of rotation measures, transverse gra-

\footnotetext{
a e-mail: wardle@brandeis.edu
}

dients of rotation measure, and circular polarization. All of these were at that time, and still now are at the forefront of AGN astrophysics and are uniquely suited to the observational capabilities of the VLBA.

In 2005 the first of a series of papers from the MOJAVE monitoring program was published on the polarization properties of a complete sample of 133 northern AGN with $15 \mathrm{GHz}$ flux densities > $1.5 \mathrm{Jy}$ ([3] et seq.). The MOJAVE program is now over 10 years old (since 2002) and has built up a huge data base of images and movies in both total intensity and linear polarization. The very comprehensive website (http://www.physics.purdue.edu/astro/MOJAVE/index.html) has become an invaluable resource for researchers, teachers and students. Presently they observe for 24 hours about 18 times per year. In one session they observe 30 sources, and the monitoring cadence on a single source is typically 2-3 months.

No other monitoring program comes close to MOJAVE in either scope or longevity. Perhaps the next most ambitious program is the imaging in total intensity and linear polarization of 36 blazars at $43 \mathrm{GHz}$ by the Boston University group (http://www.bu.edu/blazars/research.html). This is in fact a continuation of monitoring programs that started in 1994 ([4] et seq). An important feature is that the monitoring is broadband, extending up to gamma ray energies, and in the optical and infrared they also monitor the linear polarization.

The monitoring programs are important, of course, because AGN are variable. Deep observations of individual or a small number of sources, often at multiple wave- 
lengths, are beyond the reach of the large monitoring programs, and are complementary to them. Many of the participants at this conference have made such observations and many are reported in these proceedings.

The EVN is also capable of measuring linear polarization. The limited available observing time and non-uniform antenna properties result in (according to SAO/NASA ADS) 19 papers reporting linear polarization observations with the EVN compared to 480 with the VLBA. On the other hand, the large collecting areas of some of the EVN antennas can greatly improve sensitivity and $u-v$ coverage compared to the VLBA alone, e.g. [5].

Linear and circular polarization observations with a VLBI array are difficult, and questions of reliability and the proper assessment of errors are critically important. These have been investigated in detail both analytically and by extensive simulations in largely unsung appendices to observational papers e.g. [6], [7], [8], [9]. They should be read by every practitioner in the field.

\section{Fractional polarization}

It has been known from almost the earliest days of VLBI polarimetry that the parsec-scale jets of AGN can be moderately strongly polarized, the cores much less so, and that galaxies exhibit very little polarization at all compared to quasars and BL Lac objects (5 GHz, [10]). The first epoch linear polarization results on $133 \mathrm{AGN}$ at $15 \mathrm{GHz}$ from MOJAVE [3] confirm this picture and flesh it out. Table 1 shows the median fractional polarizations of cores and jets for three classes of optical identification. The numbers are estimated from the histograms in [3]. The reader is referred to that paper for the shape of the distributions.

Table 1. Median fractional polarizations at $15 \mathrm{GHz}$

\begin{tabular}{lcc}
\hline Optical ID & Cores & Jets \\
\hline BL Lacs $(\mathrm{N}=22)$ & $4.3 \%$ & $10.0 \%$ \\
Quasars $(\mathrm{N}=95)$ & $1.7 \%$ & $3.5 \%$ \\
Galaxies $(\mathrm{N}=7)$ & $<0.3 \%$ & $2.0 \%$ \\
\hline
\end{tabular}

A survey of 177 sources from the Caltech-Jodrell Flatspectrum (CJF) sample at $5 \mathrm{GHz}$ yielded similar results [11]. It should be noted that because the cores are so much brighter than the jets, they usually have a larger polarized flux density than the jets, even though their fractional polarization is lower. The VIPS survey observed 1127 flatspectrum sources at $5 \mathrm{GHz}$, but only for 500 seconds each [12]. It is not clear if this was long enough to detect polarization in many of the jets. Lister [13] observed 32 AGN from the Pearson-Readhead sample [14] at $43 \mathrm{GHz}$. Because the jets have steep spectra, he too had limited dynamic range for the jet components. They appear to be more strongly polarized than at 5 or $15 \mathrm{GHz}$ (by about a factor of 2), but this may be a selection effect due to counting only detected components. The jets are certainly Faraday thin at 15 and $43 \mathrm{GHz}$ (see below) and it is difficult to see why they should be systematically more strongly polarized at the higher frequency. When comparing two distributions that contain both detections and bounds, it may be profitable to use a method devised to deal with a similar problem at X-ray wavelengths [15].

All observers find a general increase in jet fractional polarization with increasing (projected) distance from the core. While this is almost certainly real, the precise form of the relationship is again complicated by a selection effect: because the surface brightness of jets decreases with distance from the core, only high degrees of polarization can be detected at large distances.

Because the jets are optically and Faraday thin at short centimeter wavelengths, their fractional polarization is a measure of the degree of spatial order (or lack of it) in the magnetic field on the scale of the point spread function. In a perfectly uniform magnetic field, opticallythin synchrotron radiation has a fractional polarization of $p_{0}=(3 \alpha+3) /(3 \alpha+5)$ where $\alpha$ is the spectral index $\left(S_{v} \propto v^{-\alpha}\right)$. If we describe a partially ordered field as a uniform (say, poloidal) component, $B_{p}$, plus an isotropic random component $B_{r}$, then the fractional polarization is [16]

$$
\frac{p}{p_{0}}=\frac{3 \xi^{2} \sin ^{2} \theta^{\prime}}{2+3 \xi^{2} \sin ^{2} \theta^{\prime}} \quad \text { where } \xi=B_{p} / B_{r}
$$

Here $\theta^{\prime}$ is the angle between the jet and the observer in the rest frame of the emitting fluid. So for example, if $\theta^{\prime}=\pi / 2$ and $\alpha=1$, then $p=10.5 \%$ for $B_{p} / B_{r}=0.33$. Here the net magnetic field direction is aligned with the jet, so the electric vector position angles (EVPA) will be aligned transverse to the jet direction.

Often the EVPAs are aligned parallel to the jet, indicating a net transverse magnetic field. This is often modeled as the compression of a completely disordered magnetic field by a shock [17], [18]. The resulting polarization is

$$
\frac{p}{p_{0}}=\frac{-\left(1-k^{2}\right) \sin ^{2} \theta^{\prime}}{2-\left(1-k^{2}\right) \sin ^{2} \theta^{\prime}} \quad \text { where } k \text { is the compression }
$$

Here $k$, the degree of compression, is defined as unit length being compressed to length $\mathrm{k}$. The net magnetic field is transverse to the jet, so the EVPAs are aligned parallel to the jet (in the coordinate system chosen here (jet pointing east-west), this is indicated by the minus sign (negative Stokes Q)).

The similarity between these two expressions is interesting. They are both just particular cases of a family of models describing partially ordered magnetic fields. If a completely disordered magnetic field is stretched along the jet, then $k>1$, and the EVPAs become transverse to the jet as in the first case. The difference is that the poloidal field in (1) can be vector ordered, in which case the jet has a net magnetic flux which must be conserved. In the second case with $k>1$, the jet has no net magnetic flux. This may well describe FR II jets on kpc scales where the apparent magnetic field direction tends to be aligned with the jet (e.g. [19]), even though any vector ordered poloidal field must be negligible so far from the nucleus. (Probably shear substitutes for stretching [20]).

The clear difference between the fractional polarization of BL Lac objects, quasars and galaxies can be ex- 
plained in terms of these simple models as being due to the dependence on $\theta^{\prime}$, which is related to the angle $\theta$ between the jet and the line of sight in the observer frame by the aberration formula

$$
\cos \theta^{\prime}=\frac{\cos \theta-\beta}{1-\beta \cos \theta} \quad \text { where } \beta c \text { is the fluid velocity }
$$

The point is demonstrated in table 2, which loosely mimics table 1. (We have combined BL Lac objects and high optical polarization quasars (HPQs) and called them "blazars." The categories very roughly correspond to the LVP, IVP and HVP categories discussed in [21]). We show jet fractional polarizations at various orientations to the line of sight, using equation (1) for a jet with a Lorentz factor $\Gamma=10$ and $\xi=B_{p} / B_{r}=0.33$ and $\alpha=1.0$. In the third column we also list the apparent superluminal speed (assuming the pattern and fluid speeds are equal). A relativistic jet exhibits the fastest superluminal motion when viewed at an angle $\theta=\arcsin 1 / \Gamma$. In the frame of the emitting fluid this always corresponds to $\theta^{\prime}=90^{\circ}$, and this is the angle at which the ordered part of the magnetic field is most prominent for both of the simple field models described above. Thus it is not surprising that blazars exhibit some of the higher degrees of polarization. For larger (and smaller) angles to the line of sight, $\theta^{\prime}$ deviates quickly from $90^{\circ}$ and the fractional polarization drops accordingly. In table 2, the first line corresponds to a jet that exhibits the maximum possible superluminal motion for a $\Gamma=10$ jet. Taking some license, we have designated it a "blazar." The next two lines correspond to larger angles to the line of sight, and we designate these as "quasars." In the unification of FR II galaxies and quasars proposed first by Barthel [22], AGN identified as quasars are oriented between $0^{\circ}$ and $45^{\circ}$ to the line of sight. Those AGN oriented at larger angles are identified as radio galaxies because the broad line region is obscured by a dusty torus. In table 2 we find that our model jet at $45^{\circ}$ to the line of sight has a fractional polarization of only $0.7 \%$. Jets at even larger angles to the line of sight (i.e. galaxies) will exhibit even weaker polarization. The low degree of polarization is because even at $\theta=45^{\circ}$, in the frame of the emitting fluid $\theta^{\prime}=166^{\circ}$, and the poloidal field in (1) is seen nearly endon, and the Laing sheet in (2) is seen nearly face-on.

Table 2. Simple model for jet polarization

\begin{tabular}{lcccc}
\hline Optical ID & $\theta$ & $\beta_{a p p}$ & $\theta^{\prime}$ & $p$ \\
\hline "Blazar" & $5.7^{\circ}$ & 9.9 & $90.0^{\circ}$ & $10.5 \%$ \\
"Quasar" & $15^{\circ}$ & 6.6 & $138^{\circ}$ & $5.1 \%$ \\
"Quasar" & $30^{\circ}$ & 3.6 & $159^{\circ}$ & $1.6 \%$ \\
"Galaxies" & $>45^{\circ}$ & $<2.4$ & $>166^{\circ}$ & $<0.7 \%$ \\
\hline
\end{tabular}

One of the purposes of the preceding paragraphs is to draw attention to the critical role that aberration plays in the appearance of polarized sources in relativistic motion. It is also to present a very simple model that can reproduce the essential features of the observations summarized in table 1. Depolarization by an external Faraday screen, perhaps the "dusty torus," has been invoked to account for the low fractional polarization of FR II radio galaxies on pc-scales compared to quasars and blazars (e.g. [23], [24], [25]). From the above discussion, it is not at all clear that a depolarizing screen is actually needed.

Finally (on the subject of fractional polarization) it is likely that the lower polarization of the cores compared to the jets is due to a combination of both opacity and (in some sources, at least) Faraday rotation. In the inhomogeneous jet model of Blandford \& Königl [26], the base of a jet is opaque (with the $\tau=1$ surface moving downstream from the apex with increasing wavelength). The bulk of the total intensity emission comes from the vicinity of the $\tau=1$ surface. Opacity severely depresses fractional polarization so the bulk of the polarized emission comes from somewhat downstream from the $\tau=1$ surface where the jet is intrinsically fainter. The net effect is that the fractional polarization of a Blandford - Königl core is smaller by a factor of 2 or so than that of the optically thin jet, even if the magnetic field geometries are the same. This is consistent with the observational data in table 1.

\section{The alignment (or lack thereof) of EVPAs with the jet direction}

Numerical simulations and analytic models invariably assume cylindrical symmetry. It follows that the expected alignment of the EVPAs is either parallel or perpendicular to the jet. For the cores, there is a modest tendency for the EVPAs to be parallel to the inner jet direction at both 15 $\mathrm{GHz}$ [3] and $43 \mathrm{GHz}$ [13]. This is a somewhat stronger effect in BL Lac objects than in quasars, and indicates a mild preference for transverse field order, perhaps indicating shocks.

For the jet components, there is surprisingly little alignment of EVPAs with the jet direction at $15 \mathrm{GHz}$ for quasars. For BL Lac objects there is moderate alignment, similar to that exhibited by the cores. At $43 \mathrm{GHz}$, the quasars are significantly better aligned than at $15 \mathrm{GHz}$ : the median difference between the EVPA and the jet direction is about $20^{\circ}$, only a little larger than for BL Lac objects.

The general lack of alignment in the jets is somewhat puzzling, even though there are many factors that might contribute to misalignment. Several points can be made. (1) While Faraday rotation certainly occurs in compact radio sources, especially in and near the cores (see below), large values of Rotation Measure (RM) are actually quite rare. Hovatta et al [8] found that $80 \%$ of the jets in the MOJAVE sample exhibit $|\mathrm{RM}| \leq 400 \mathrm{rad} \mathrm{m}^{-2}$ which corresponds to an EVPA rotation at $15 \mathrm{GHz}$ of less than $10^{\circ}$. This is insufficient to cause the apparent lack of alignment in most sources.

(2) Oblique shocks: for a single oblique shocks the jet must be refracted (bent) because the jump conditions for the velocity components parallel and perpendicular to the shock are different [27], [28]. Where this appears to be the case (e.g. M87, [29]) useful physical parameters can be derived from the images. But if the jet has strict cylindrical symmetry, X-shaped conical shocks form, as are revealed in numerical simulations (e.g. [30]), and unless the X-shaped structure is resolved (it never has been) 
the net polarization EVPAs will still be either parallel or perpendicular to the jet.

However, most MOJAVE sources are not straight. The bends are greatly exaggerated by projection and are intrinsically quite small. But if a jet is bent by an external pressure or density gradient, for instance, then an oblique shock will form and the polarization angle will be misaligned with the jet (e.g. [31], [32]). This may well be the origin of much of the observed misalignment. The histograms of EVPA 'misalignment' in the literature do not usually differentiate between bent and straight jets. A superficial inspection of the MOJAVE data suggests that in the straighter jets, the EVPAs may be better aligned with the jet direction (both parallel and perpendicular), which lends support to the above picture. It might be extremely informative to compare the observed EVPAs with the local vector proper motion direction rather than the overall orientation of the jet.

(3) For an optically thin helical magnetic field, cancelation between the back and the front of the jet leads to EVPAs aligned with the jet if the pitch angle is greater than $45^{\circ}$, and EVPAs aligned transverse to the jet if the pitch angle is less than $45^{\circ}$. Opacity can break the strict cancellation between the front and back of the jet, but will also strongly suppress the resulting fractional polarization. Another way to break the strict cancellation is by differential Doppler boosting in a conical jet. The near side of a conical jet makes a smaller angle to the line of sight than the far side, so their Doppler factors, $D=[\gamma(1-\beta \cos \theta)]^{-1}$ are different. The boost in brightness is proportional to $D^{2+\alpha}$, and the change in this between the back and the front of the jet is sufficient to lead to a large twist in EVPAs while maintaining a high fractional polarization. This has been worked out in some detail (albeit with a somewhat idealized field model) for the arcsecond scale jet of the quasar 3C345, which exhibits such behavior [33]. A somewhat similar version of this idea was proposed in [13] (see Figure 41). In this version, the helix denotes the streamlines for the flow, which obviates the need for a conical jet.

Probably all of the above effects contribute in varying degrees to the observed EVPA misalignments, with oblique shocks connected with jet bending being the most important. Closest to the base of the jet (i.e. in the unresolved cores), Faraday rotation plays an increasingly important role (see section 5).

\section{Optical polarization and the radio-optical EVPA alignment}

When linear polarization is observed at optical wavelengths, the emission mechanism is almost certainly synchrotron radiation, and Faraday rotation can safely be assumed to be negligible. The optical EVPA obviously reflects the local field orientation where the optical emission originates. If the optical emission and the radio emission both originate from the same physical region (which is often not easy to establish), then the radio EVPA should approach the optical EVPA at the shortest radio wavelengths. This appears to be the case for a sample of 12 blazars (mostly BL Lac objects) observed at $15 \mathrm{GHz}, 22 \mathrm{GHz}$ and $43 \mathrm{GHz}$ [34]. At $15 \mathrm{GHz}$ there is no radio-optical alignment at all. At $22 \mathrm{GHz}$, the median difference in EVPA is $20^{\circ}$; at $43 \mathrm{GHz}$ it is $10^{\circ}$, and if the radio EVPAs are corrected for apparent Faraday rotation, the alignment becomes very slightly better. Similar results were found for a sample of 15 superluminal AGN observed at optical wavelengths and also at $1 \mathrm{~mm}, 3 \mathrm{~mm}$ and $7 \mathrm{~mm}$ [21].

Similar EVPAs are a necessary but not sufficient condition to establish that the emitting regions of the optical emission and the high frequency radio emission are co-spatial. Correlated variations in the EVPAs, however, would be strong evidence that the optical and radio emission come from the same part of the jet. This appears to be the case for PKS 0420-014 [35], where the optical EVPA and the $43 \mathrm{GHz}$ core EVPA both swung through about $80^{\circ}$ in the span of 9 days in late 2005, while the difference in EVPAs was always less than $15^{\circ}$. Similar behavior for PKS $0836+710$ is reported by Jorstad at this conference. While the radio and optical EVPAs do not generally track each other closely, Troitskiy (poster 34, this meeting) reports further such episodes in PKS 0420-014.

In many ways, even more interesting is that episodes of rapid swing in the optical EVPAs (tens of degrees per day, for a week or two) seem to presage two extraordinary events: (i) a huge and short-lived gamma ray outburst, and (ii) the emergence of a new superluminal component from the radio core. This is well documented for BL Lac [36], for PKS 1510-089 (the 2009 outburst in [37], and the 2011 outburst in [38], and this meeting) and also for 3C454.3 (Marscher, this meeting) and PKS 0716+710 [39]. The fact that there is not an abundance of reports of such episodes does not mean they are rare - it is entirely possible that all sources behave in this way; rather, it is a testament to the huge multi-wavelength close-spaced monitoring effort on many instruments that is required to demonstrate such correlated behavior.

The observational behavior described in the previous paragraph has led to a theoretical picture [36], [37], in which the gamma-ray outburst, the new superluminal component, and the polarized optical emission all originate far downstream (several parsecs) from the black hole at a stationary shock that accelerates the synchrotron emitting electrons. This is the location of the observed 'core' at millimeter wavelengths, but is well downstream from the beginning of the jet. That the gamma rays originate so far from the black hole is still controversial (e.g. Ghisellini, this conference). In Marscher's picture (following [40]) the rotating EVPA of the optical emission is ascribed to a synchrotron emitting blob following (low pitch angle) helical streamlines in a helical (high pitch angle) magnetic field, upstream from the stationary shock. This is suggested by a model for the magnetic launching and acceleration of jets described in [41] and [42].

In [39] a theoretical model based on Marscher's picture is fit to the observations of PKS 0716+710. The fit to the R-band total intensity and the R-band EVPA versus time are quite successful. 


\section{Rotation Measures}

The pioneering measurements of Rotation Measure (RM) on milliarcsecond scales were made by Taylor and Zavala ([43], [44], [45] et seq.) using the VLBA at several frequencies between 5 and $15 \mathrm{GHz}$. These established that large RMs of order $1000 \mathrm{rad} \mathrm{m}^{-2}$ are common in the core regions of AGN. In the jets, the RMs are more modest, rarely more than a few hundred $\mathrm{rad} \mathrm{m}^{-2}$. Such a large RM gradient over just a few milliarcseconds means that the Faraday rotation must be taking place at the source possibly internal to the jet, or in the immediate surroundings of the jet, or in the narrow line region. If the Faraday rotating screen is located near or in the source, then the observed values should be multiplied by $(1+z)^{2}$, where $z$ is the redshift, to give the RM that would be observed in the rest frame of the AGN. In [45] it was also established that RMs on milliarcsecond scales can be variable on time scales as short as a year or so. They reported large changes (in excess of $1000 \mathrm{rad} \mathrm{m}^{-2}$ ) in the core RMs for $3 \mathrm{C} 273$ and $3 \mathrm{C} 279$, even changing sign in the case of $3 \mathrm{C} 273$. It is probable that RM variability is widespread, which makes it hard to check these difficult measurements against other observations. Zavala \& Taylor attributed the variability to the motion of jet components behind a random Faraday screen in the narrow line region.

In Hovatta et al [8] this picture is largely confirmed with the much larger MOJAVE sample observing at four frequencies between 8 and $15 \mathrm{GHz}$, and measuring RMs in 149 out of 191 sources. They pay careful attention to errors, with a large fraction of the paper devoted to analysis and simulations to understand the errors. When a quantity such as rotation measure is displayed as a color image (often superimposed on the total intensity contours), the reader has no idea how large the errors are or how they vary over the image. This is solved in [8] by displaying a second image that gives the errors in the rotation measures (also superimposed on the total intensity contours). It takes twice as much space but is extremely useful, and could be emulated to good effect whenever quantitative information is displayed as false-color (e.g. spectral index maps).

The MOJAVE group find that quasars exhibit larger RMs than BL Lac objects, and that cores exhibit larger RMs than jets. However, RMs as large as $1000 \mathrm{rad} \mathrm{m}^{-2}$ are in fact rare, even in the cores. Taylor's early results indicated that most cores had RMs in excess of $1000 \mathrm{rad}$ $\mathrm{m}^{-2}$, but this seems to be an accident of small number statistics and rotation measure variability. The median rotation measures in the MOJAVE sample are $200 \mathrm{rad} \mathrm{m}^{-2}$ for quasar core regions, $105 \mathrm{rad} \mathrm{m}^{-2}$ for BL Lac core regions, $141 \mathrm{rad} \mathrm{m}^{-2}$ for quasar jets, and $71 \mathrm{rad} \mathrm{m}^{-2}$ for $\mathrm{BL}$ Lac jets. The differences between the optical subclasses are not particularly large, but they are all significant. The small tail of very high RMs consists entirely of quasars. For quasars there is also a significant negative correlation between rotation measure and de-projected distance from the core.

Whether Faraday rotation is internal or external, depolarization is normally expected at longer wavelengths.
This was studied in [8] for "isolated" jet components (those that are far enough from the core to be unaffected by opacity or core emission). At least two sources (3C273 and 3C454.3) exhibited inverse depolarization where the fractional polarization increases at longer wavelengths. This is very difficult to produce with an external screen, and indicates that internal Faraday rotation is occurring. This is investigated in detail in [46]. For the other sources, the connection between depolarization and rotation measure can be modeled with simulations to determine the statistical properties of the Faraday screen. Hovatta et al found that an external, random Faraday screen viewed through just a few lines of sight was consistent with the observations. It should be noted that inverse depolarization is not the only indicator of internal Faraday rotation. For instance, circular polarization observations of 3C279 [82] strongly indicate the presence of some internal Faraday rotation in order to drive the Faraday conversion that generates the observed circular polarization. It is likely that some internal Faraday rotation (by low energy relativistic electrons) is present in most sources in which circular polarization is detected, and perhaps in all sources at some level.

Finally, the MOJAVE group detected a significant transverse rotation measure gradient in only four sources (4C39.25, 3C273, CTA 102, and 3C454.3). These are discussed further below. It is not obvious at present if RM gradients are not very common, or if they are ubiquitous but can only be detected with confidence in the brightest sources.

At higher frequencies (i.e. when observations at 22, 43 and $86 \mathrm{GHz}$ are made), core RMs are found to be dramatically larger. Another way of saying this is that a plot of EVPA against wavelength squared is very non-linear. It was proposed in [47] that $|\mathrm{RM}| \propto v^{a}$ with $a$ varying in the range $1-4$ for the six objects observed. In [21], [48] and [49], further evidence is given of much higher RMs at higher frequencies. For instance, in [49], reporting observations of $40 \mathrm{AGNs}$ at 15, 22 and $43 \mathrm{GHz}$, it was found that (of 34 measured RMs) 59\% had values exceeding $1000 \mathrm{rad} \mathrm{m}^{-2}$ (compared to only a few percent in the MOJAVE sample), and $18 \%$ had values of $|\mathrm{RM}|$ in excess of $10,000 \mathrm{rad} \mathrm{m}^{-2}$.

That RMs are larger at high frequencies is not altogether unexpected. In the well-known inhomogeneous relativistic jet model of Blandford and Königl [26], the jet is brightest where the optical depth is close to unity, and this region is seen as the core in milliarcsecond resolution images. In an expanding jet, the electron density and the magnetic field both decrease with distance from the apex and the location of the $\tau=1$ surface, and hence the apparent radio core, moves down the jet with increasing wavelength. For the particular parameters chosen in [26], the distance of the core from the apex is proportional to the wavelength of observation. A more general treatment is given in [50]. With careful registration of images made at different wavelengths, this 'core shift' can be measured (e.g. [51], [52]) and be used to determine certain physical parameters, such as $B_{1}$, the magnetic field strength at $1 \mathrm{pc}$. 
The changing core position means that at different wavelengths the lines of sight to the core are different. At shorter wavelengths the core is closer to the base of the jet, and it is entirely plausible that the line of sight traverses denser and more strongly magnetized plasma, leading to larger rotation measures, as observed. This explanation requires, of course, that the Faraday screen is very close to the jet. According to [51] the typical displacement of the core at $\lambda 4 \mathrm{~cm}$ from the optical core is 0.1 mas. The Faraday screen must vary substantially on scales less than this, i.e. on sub-parsec scales at the source.

There is a second consequence of the Blandford Königl model that can complicate the analysis of Faraday rotation in compact jets. This concerns internal Faraday rotation. Because the position of the radio core moves down the jet with increasing wavelength, and both the magnetic field and the electron density decrease with distance, internal Faraday rotation can be independent of wavelength! This leads to the odd situation where a plot of EVPA against $\lambda^{2}$ can be flat even though there is substantial internal Faraday depth at every wavelength. Detailed modeling of this effect has not been done, so it is unclear if it might explain some of the misaligned core EVPAs.

If internal Faraday rotation is present, then the electrons responsible are most likely those at the low energy end of the relativistic electron distribution [53], [54]. Measurements of internal Faraday depth therefore constrain the shape of the distribution at low energies, which is difficult to observe directly, but is needed for calculating, for example, jet energetics, or equipartition magnetic fields.

Two other practical complications should be mentioned here. The first is the well-known fact that EVPAs are ambiguous to within arbitrary additions of $\pm \pi$ radians. This is more important at longer wavelengths where some authors have added several times $\pi$ radians to their measurements in pursuit of a linear EVPA versus $\lambda^{2}$ graph, even if there is little other evidence to support the high resulting RMs. At the short centimeter wavelengths where nearly all of the RM measurements with the VLBA have been made, the " $\pi$ ambiguity problem" becomes somewhat subtle. For instance, a $\pi$ radian ambiguity in EVPA between $8 \mathrm{GHz}$ and $15 \mathrm{GHz}$ corresponds to a change in apparent RM of about $2600 \mathrm{rad} \mathrm{m}^{-2}$, and this is larger than any RM measured in [8] for isolated jet components and is unlikely to be appropriate. For core components, such high RMs are seen, but RMs may be frequency dependent for the reasons discussed above, and the third frequency (e.g. $22 \mathrm{GHz}$ ) may not entirely resolve the ambiguity. As a practical matter, the $8 \mathrm{GHz}$ and $15 \mathrm{GHz}$ bands are usually split into two or more separate sub-bands. The EVPA gradients between these sub-bands can be critical in establishing whether shifting all the EVPA measurements within a band by $n \pi$ radians is appropriate or not.

The second practical complication is that the antennas of the VLBA are fixed, unlike those of the VLA, so it is not possible to make "scaled-array" observations with similar resolution at different frequencies. What is often done is to convolve the higher frequency images down to the resolution of the lowest frequency image. This is not entirely satisfactory because the higher frequency images lack the short interferometer spacings that are present in the low frequency image. Care must be taken in interpreting sources with complex RM structure, especially if there is also spectral index structure.

When a source is bright and well-resolved, RM images can reveal much interesting and important detail. A short but rich paper by Gómez et al. [55] illustrates this for the source $3 \mathrm{C} 120$. They present 11 epochs of total intensity and polarization images made at 15, 22 and $43 \mathrm{GHz}$. The jet, which contains several superluminal components, can be followed to at least 10 mas ( 30 beamwidths) from the core, and it is resolved in the transverse direction by $2-3$ beamwidths. The resulting maps of fractional polarization, RM and intrinsic (zero wavelength) EVPA contain a wealth of detail.

These include (1) the fractional polarization increases along the jet from essentially zero at the core to nearly $20 \%$ at 10 mas. Between 3 and 5 mas the fractional polarization dips and this coincides with a local region of very high $\mathrm{RM}\left(\sim 6000 \mathrm{rad} \mathrm{m}^{-2}\right)$. They plausibly attribute this to the interaction of the jet with a local cloud. (2) Transverse to the jet direction, the fractional polarization is noticeably asymmetric, reaching $25 \%$ at the northern edge, only $15 \%$ at the southern edge, and drops smoothly to $\sim 5 \%$ in the middle. All three frequencies give similar profiles, so the low polarization in the middle is due to magnetic field structure and not to Faraday depolarization. This means that the line of sight passes through regions of nearly orthogonal magnetic field, or that the field in the spine of the jet is largely disordered. (3) The EVPA corrected for Faraday rotation is everywhere transverse to the jet direction, meaning that the magnetic field is dominated by the poloidal component. (4) There is a clear transverse gradient in RM. At a distance of 4 mas from the core, the RM varies monotonically from $\sim 1800 \mathrm{rad} \mathrm{m}^{-2}$ on the northern side to $\sim 4000 \mathrm{rad} \mathrm{m}^{-2}$ on the southern side. It should be noted that while all of the above features can be seen to a greater or lesser extent in the individual images, they are most clearly seen when the images from different epochs are averaged together. Although this tends to smear out the moving features, it increases the SNR and brings out the underlying source properties. This technique might be used to advantage with other sources that have been observed at multiple epochs.

Another source that is both bright and well-resolved is $3 \mathrm{C} 273$. This was the first source in which a transverse gradient in Faraday rotation was observed [56] and we discuss it in the next section.

\section{Rotation Measure gradients}

The detection of a RM gradient transverse to the jet in 3C273 by Asada et al. [56] was confirmed in [57] and [58]. (Transverse RM gradients had been suggested by Blandford [59] as a way of detecting the toroidal component of magnetic fields that might play an important role in collimating jets.) Many claimed detections of transverse RM gradients in other sources then ensued. In [60] 29 sources 
from the literature were listed that were claimed to exhibit transverse RM gradients on pc-scales. This prompted Taylor \& Zavala [61] to suggest that many of these claims were premature. Among their criticisms were that (1) most of the jets were inadequately resolved in the transverse direction, (2) that the apparent gradient had inadequate signal to noise, (3) that the source was not optically thin at the location of the gradient, i.e. it was too close to the core or actually was the core, and (4) that the gradient was not monotonically smooth across the source. Similar cautions were expressed in [62].

As regards point (1), Taylor and Zavala suggest that at least three beamwidths across the source are required. This point has now been extensively investigated with Monte-Carlo simulations by Hovatta et al. [8], and also by Gabuzda and co-workers [63], [9], [64]. Hovatta et al. emphasize that thermal noise can generate high RMs and spurious gradients if the jet is less than two beams wide in polarization. But they consider the Taylor-Zavala criterion to be overly cautious, and that a jet that is only two beams wide will give a reliable gradient if the SNR exceeds $3 \sigma$ (considerable attention must be paid to calculating the errors properly). They also state "Attempting to detect RM gradients in jets less than two beams wide is discouraged as the probability to detect false positives exceeds 0.02 even when a $3 \sigma$ limit is used." This statement is not quite in keeping with their own Figure 30, which, as quoted in [9] and [64], seems to indicate that for a $3 \sigma$ limit, the false positive rate is only 0.01 even at one and a half jet beams. Hovatta (private communication) has confirmed that the figure is correct and that the "0.02" in the text was a typographical error, and also points out that a $1 \%$ false positive rate becomes more significant in the context of large surveys like MOJAVE where well over 100 sources were observed.

Murphy et al [64] and Mahmud et al. [9] demonstrated through Monte-Carlo simulations that a RM gradient in a jet whose width is even somewhat smaller than the beam can still be reliably detected, even though the magnitude of the gradient is reduced. Presumably this result must depend on the SNR, though that dependence is not quite clear in these papers.

Taylor and Zavala's third point has some merit. Hovatta et al found that if they considered only "isolated" jet components (i.e. components sufficiently separated from the core or other bright components that their polarization is not affected by their proximity to other features) then the histogram of jet component RMs narrowed and lost its high RM tail. The implication is that measurements made close to the core may be affected by the core. Transverse gradients detected for the core itself should certainly be viewed with skepticism. If the core is unresolved in total intensity, then any gradient in polarization must surely be due to imaging errors such as residual D term errors [6].

There is another source of error that may not have been taken account of in the simulations. For the most part (see the previous section) RM measurements are consistent with a random Faraday screen close to the jet, with a correlation length somewhat smaller than a typical beam width. If the screen has a variance in RM of $\sigma^{2}$ then this should probably be added to the uncertainty in the apparent RM gradient. For example, if the jet is only two beams wide, then an RM gradient of order $\sigma$ /beamwidth will inevitably be measured, but this merely reflects the two point correlation function of the Faraday screen, and is not necessarily evidence for a toroidal magnetic field. If such a screen exists, then Taylor \& Zavala's fourth point is probably not correct because the screen could add fluctuations that disrupt both the smoothness and the monotonic behavior of the gradient.

The above discussion is not intended to cast doubt on the published RM gradients so much as to urge caution. A proper error analysis is complex, and sometimes the Scottish verdict of "not proven" may be more appropriate. Also, in almost every case where transverse gradients have been detected, only one cut shows a significant gradient. For a true helical magnetic field, one might expect to see a series of cuts down the jet that exhibit transverse gradients. Limited sensitivity probably accounts for the lack of such cases, so much deeper observations of a few sources which display a single RM gradient would be of great interest. If the RM gradient continues down the jet or if it does not, would be revealing either way.

The source for which a transverse RM gradient seems irrefutable is 3C273. In [8] (see their Figure 8) the gradient can be seen in 3 transverse cuts, the last being about 12 mas from the $\mathrm{c}$ ore. The magnitude of the gradient (very plausibly) decreases with distance from the core, and is similar in March and June 2009 except near the core (note the erratum in [65]). Others have also seen the gradient in multiple cuts down the jet (e.g. [66], [67], both detecting the gradient out to 20 mas). The largest gradient that has been observed to date in this (or any other) source was seen at 0.9 mas from the core by Attridge et al [58], using VLBA observations at 43 at $86 \mathrm{GHz}$. The observed gradient was $1.3 \times 10^{5} \mathrm{rad} \mathrm{m}^{-2} \mathrm{mas}^{-1}$. Comparing this to the gradients measured at about 9 mas reported in [56], [57], and [8] the transverse gradient decreases with core distance (the "gradient of the gradient") very roughly as (core distance $)^{-3}$.

Just as single RMs, especially in the cores, exhibit variability (see above), so apparently, do transverse RM gradients. Asada et al [67] report such variations for 3C273: it is difficult to quote values for the change in gradient because the shape changed between 1995 and 2002 , but over this period the gradient became significantly steeper. Hovatta et al [8] also see changes over only 3 months, especially near the core. Asada et al use the variability to rule out the narrow line region as a source for the RM gradient, and attribute it to a helical magnetic field in a sheath surrounding the jet. Hovatta et al saw changes over a much shorter time span, over which the component had not measurably moved, and therefore attributed the variability to internal Faraday rotation. However, Gómez et al. [68] in a further analysis of $3 \mathrm{C} 120$, found a lack of correlation between changes in polarization and changes in RM, indicating that the emitting jet and the source of Faraday rotation are not closely connected. They also argue, based 
on a region of enhanced RM that has remained constant over at least 3 years, that a significant fraction of the RM originates in foreground clouds. Taken altogether, there is now plausible evidence for some Faraday rotation internal to the jet, some in a physically-connected sheath around the jet, some nearby but not physically connected with the jet, and some in more distant clouds such as the narrowline region. Individual sources may not exhibit Faraday rotation in all four regions, but the contributions of each will be difficult to disentangle.

Finally, Mahmud et al [69] report a most surprising result for the BL Lac object B1803+784, where the direction of the RM gradient apparently reverses between observations made two years apart. The explanation favored by the authors involves nested helical fields of opposite handedness, deriving from oppositely directed currents in the inner and outer parts of the accretion disk. This is based directly on the "magnetic tower" model of Lynden-Bell [70]. The idea is further developed in [9]. However, Broderick \& McKinney [62] criticize this model both on physical grounds, and because their GRMHD simulations reveal no such structures.

The results of this and the previous section have demonstrated that there is a wealth of important information in the Faraday rotation images. But too many of them suffer from not-quite-enough resolution and notquite-enough sensitivity, and this is the cause of much of the skepticism and disagreement found in the literature. This need not be the case. The new high bandwidth receivers on the VLBA mean that the sensitivity at all frequencies is greatly improved. One take-away from this review should be (1) that one should observe at the highest frequencies possible, since the resolution on a rotation measure map is set by the beam-width at the lowest frequency used, and (2) integration times must be long enough that the polarized emission of relatively faint structure is well imaged with sufficient SNR that reliable rotation measures can be derived. Of course, since the jets have steep spectra, these two points are not entirely compatible with each other. But it is far better (for these purposes) to image a few objects very deeply than to survey many sources at moderate SNR.

\section{Helical or toroidal magnetic fields?}

A transverse gradient of RM indicates a toroidal component of magnetic field (subject to the caveats expressed above). Often in the literature that is taken to mean that the magnetic field is a helix. Whether or not that is actually the case merits discussion.

A helical field is a combination of a toroidal field, $B_{\phi}$, and a poloidal field, $B_{\theta}$, both of which are vector-ordered. The RM gradient only establishes the toroidal part, and there are several considerations that make a large-scale helical field problematic.

First, if the jet expands conserving magnetic flux, we expect $B_{\phi} \propto R^{-1}$ and $B_{\theta} \propto R^{-2}$, where $R$ is the jet radius. This means that the pitch angle of the helix increases and the jet is soon dominated by the toroidal component.
Observed opening angles can be some tens of degrees, but these, of course, are seen in projection. Typical deprojected opening angles are much smaller, mostly in the range $1^{\circ}$ to $3^{\circ}[71]$, but this is still sufficient for the toroidal field to dominate fairly quickly. It should be noted that many theoretical papers work "in the cylindrical approximation," and therefore do not include this effect.

Second, although the synchrotron emission from a helical magnetic field is symmetrical about the jet axis when the line of sight is perpendicular to that axis in the frame of the emitting fluid, from any other viewing angle there are transverse asymmetries in total intensity and especially polarization that should be observable. This was first pointed out by Laing [72] and was studied in some detail in [73]. The perpendicular case corresponds to $\theta \approx \sin \theta=1 / \Gamma$ (see section 2), but the most probable angle to the line of sight in a flux-limited sample of sources selected by their Doppler boosted emission is given in [74] as $\theta \approx 1 / 2 \Gamma$, so we would generally expect to see the predicted asymmetries in jets containing helical magnetic fields. A variety of asymmetries in the RM gradient (and presumably other source properties) can also be produced in a model discussed in [75], which invokes relativistic helical motion in the jet.

In $3 \mathrm{C} 273$, the source with the most secure detection of transverse RM gradients, there are indeed asymmetries between the north and south sides of the jet in both total intensity and polarization. These could be the predicted asymmetries of a helical magnetic field, but unfortunately there are other asymmetries present too. The jet is seen to follow a gently curving trajectory on the sky; the intrinsic curvature must be quite small, but any bending is likely to lead to compression on one side of the bend and rarefaction on the other, and the synchrotron emissivity and the magnetic field topology are both sensitive to these. Also, kinematic analysis of component motions reveals complicated patterns of accelerations [76], changing position angles of ejection [77] and possible precessional motion [78]. The asymmetries due to a possible helical magnetic field may be difficult to disentangle from those due to other causes.

Third, it is striking that in many of the best observed jets, the RM corrected EVPAs are nearly everywhere perpendicular to the local jet direction. Examples are 3C273, 3C120, CTA102 and 3C454.3 (after the bend). This implies that the dominant magnetic field direction is parallel to the jet, whereas a helical field in an expanding jet should quickly increase in pitch angle, resulting in a dominant magnetic field direction that is perpendicular to the jet.

The decay of the vector-ordered part of the poloidal field as the jet expands seems unavoidable. This suggests that the observed component of the magnetic field parallel to the jet is not, for the most part, vector-ordered, and instead consists of, for example, sheared loops of field or sheared tangled field (e.g. [20], [79], [80], [81]). Such fields carry zero net magnetic flux, so they do not necessarily decay as $1 / R^{2}$. It is possible to constrain the degree of vector-ordering of the magnetic field by measur- 
ing circular polarization. This was done for the quasar $3 \mathrm{c} 279$ in [82]. It is also interesting that a toroidal field plus sheared loops of poloidal field does not exhibit the transverse asymmetries of a vector-ordered helical field, whatever the viewing angle. The total intensity and polarization profiles of such a magnetic field model has been worked out by Mizrahi [83] and applied to 3C273. They will be published shortly.

\section{Circular polarization}

Circular polarization observations of AGN are challenging carry out because the signals are small and instrumental effects can be large. Ideally one would like to use orthogonal linearly polarized feeds to measure circular polarization. Those are the feed polarizations at both Westerbork and the Australian Telescope Compact Array (ATCA), but neither array has the resolution to image pc-scale jets. The VLBA uses circularly polarized feeds, which is ideal for observing linear polarization, but less so for observing circular polarization.

Homan \& Wardle [84] described in detail three different ways of making circular polarization images with the VLBA to mitigate the problem of using circular feeds. In practice, the "gain-transfer" technique has proven to be most successful, provided that a large enough number of sources are observed. For instance, in [85], 40 AGN were observed and circular polarization was detected in 11 of them, with the other 29 sources serving, pro tem, as negligibly polarized calibrators for following the variations in the right- and left-hand gains. (At present there are no known AGN with reliably zero or constant circular polarization that could be used for this purpose.) Using these techniques, $3 \sigma$ detections as low as $V / I=0.15 \%$ were achieved. Results from that survey included a possibly surprising lack of correlation between linear polarization and circular polarization levels, and a suggestion (5 out of 6 sources) of long-term stability in the sign of the circular polarization when compared to observations made some 20 years earlier by Komesaroff et al. [86].

Using ATCA, where considerable design attention has been paid to detecting circular polarization, Rayner, Norris \& Sault [87] achieved astonishing $3 \sigma$ levels as low as $V / I=0.03 \%$. They found that quasars and BL Lac objects have systematically higher circular polarization than radio galaxies, and that the highest levels of circular polarization are associated with total-intensity variability and flat or inverted spectral indices, indicating that circular polarization is associated with blazar activity. The connection to source compactness was manifested in [88] which reported levels of circular polarization as high as nearly $4 \%$ in the scintillating component of the intraday variable PKS 1519-273. This prompted Marquart \& Melrose [89] to propose an ingenious mechanism for producing circular polarization based on refractive scintillation. While this mechanism may apply to intra-day variables such as PKS 1519-273 (and other ultra-compact sources such as Sgr A*), it predicts zero mean fractional polarization, with very short time-scales (hours) for switching the sign of the polarization. This seems incompatible with the observations of most AGN.

One epoch of MOJAVE observations (at $15 \mathrm{GHz}$ ) has been analyzed for circular polarization [7]. They detected "strong circular polarization" ( $\geq 0.3 \%)$ in about $15 \%$ of their sample of 133 sources. The level of polarization was typically in the range 0.3 to $0.5 \%$ of the local Stokes I. The circular polarization was mainly associated with the core components, but several strong jets were also detected, such as $3 \mathrm{C} 84$, where local fractional polarization reached $2 \%$. In $3 \mathrm{C} 273$, several circularly polarized components could be seen, with the degree of polarization decreasing down the jet. Comparing their results to the $5 \mathrm{GHz}$ results in [85], for 8 out of 10 sources the fractional polarization at $15 \mathrm{GHz}$ was higher than at $5 \mathrm{GHz}$, though not necessarily with the same sign. They found no strong correlations between fractional circular polarization of jet cores with source type, redshift, EGRET detections, or linear polarization.

Gabuzda and her co-workers have also made circular polarization observations of a substantial number of sources at several frequencies with the VLBA ([90] at 15 $\mathrm{GHz}$; [91] at 15, 22 and $43 \mathrm{GHz}$ ). In the latter paper they compare their $15 \mathrm{GHz}$ results with those from MOJAVE. For the 16 sources in common, there is no disagreement in sign, and differences in magnitude are entirely accounted for by modest variability. This is extremely encouraging considering how challenging these measurements are, and it strongly suggests that these small fractional polarizations, when measured by experienced observers, are real and contain real physical information. At $22 \mathrm{GHz}$, they find that the fractional polarization is equally likely to be larger or smaller than at $15 \mathrm{GHz}$, while at $43 \mathrm{GHz}$ it is in all cases larger than at either lower frequency. This is important because it seems to run counter to the expectations from either of the two main mechanisms for producing circular polarization - the intrinsic circular polarization of synchrotron radiation [92], or the Faraday conversion of linear polarization to circular polarization [54]. Both mechanisms predict stronger circular polarization at lower frequencies in simple, optically-thin models. But the flat or rising spectrum of fractional circular polarization towards shorter wavelengths can be understood straightforwardly in inhomgeneous jet models, where the moving location of the $\tau=1$ surface with wavelength has strong effects on the predicted spectra of all four Stokes parameters [93].

In common with other observers, Gabuzda also finds consistency in the sign of circular polarization across epochs separated by several years or more. This is important because this time-scale is much longer than the typical time-scale for blazar activity, which is of the order of a year or less. This implies that the circular polarization observations are measuring a fundamental and long-lived property of the AGN. This might be the spin of the black hole [94] or a small residual component of a vector ordered poloidal field.

Another source of information concerning the circular polarization properties of AGN has been the observations 
made by the University of Michigan group [95]. Using a single $26 \mathrm{~m}$ dish, they measured the circular polarization of strong sources at $8 \mathrm{GHz}$ from 1978 to 1984 , and then (at 5, 8 and $15 \mathrm{GHz}$ ) from 2001 to 2012, when the telescope was shut down for lack of funding. What the Michigan telescope lacked in sensitivity and resolution, it made up for in frequency of monitoring and excellence of calibration. The data are freely available (the entire data base must contain more than a million measurements) and are an invaluable data base on bright AGN. They broadly confirm what the more sporadic VLBA observations find: that the circular polarization is variable in amplitude but relatively stable in sign. In general the sign of the circular polarization was the same at all three frequencies, but sometimes it was not. This is easier to understand in the context of Faraday conversion than the intrinsic mechanism.

The promise of circular polarization measurements is that they may permit, depending on the emission mechanism, direct measurement of the magnetic field strength, its polarity, the true magnetic flux carried by a jet (and hence the flux generated at the central engine), the low energy cut-off in the relativistic electron spectrum, and the composition of the radiating plasma - whether it is predominantly an electron-proton plasma or an electronpositron plasma, or a mixture of the two [96]. None of these can be determined directly from measurements of the first three Stokes parameters (I, Q, U). Observations of Stokes V therefore offer a new way of measuring fundamental physical properties of AGN (and also of the smaller-scale jets in Galactic X-ray binaries).

The two most often considered mechanisms for generating circular polarization are the small intrinsic circular polarization of synchrotron radiation, and the less familiar but probably more important effect due to linear birefringence called Faraday conversion. The basic expressions for all the relevant emission and absorption and conversion coefficients can all be found in [54]. Practical formulae that can be applied to optically thin sources with various magnetic field configurations can be found in [93]. But the strongest circular polarization is likely to be generated near the $\tau \approx 1$ surface [97] and practical expressions for this partially opaque regime have not been derived. To model the observations of real sources with partially ordered fields, it is necessary to carry out Monte-Carlo simulations. In these, the analytic solutions of the full coupled equations of transfer for all four Stokes parameters given in [54] can be applied cell by cell along each line of sight within the point spread function. The main variables include the low energy cut-off of the electron energy spectrum, a complete model for the magnetic field and its vector ordering, and the normalized lepton number (relative numbers of electrons and positrons). This is a large parameter space, so it is very important to model the observed linear polarization at the same time. This greatly cuts down on the number of models that might otherwise explain the circular polarization observations.

It is worth noting some contrasting features of the two mechanisms. The intrinsic mechanism depends on the vector ordered part of the magnetic field and on the lepton number in the same way as Faraday rotation does. Thus a pair plasma cannot generate either intrinsic circular polarization or (internal) Faraday rotation. The same is true if the magnetic field contains many reversals of direction. Faraday conversion, on the other hand, is independent of the sign of the electron charges and also of the direction of the magnetic field. So a pair plasma in a highly sheared field can generate circular polarization by Faraday conversion. However, conversion operates on Stokes $\mathrm{U}$. (This is in a reference frame where the local magnetic field defines the direction Stokes $-\mathrm{Q}$ (and therefore radiates Stokes $+Q)$ ). There are several ways of generating some Stokes U: (1) internal Faraday rotation, (2) stochastically, in a somewhat disordered magnetic field, or (3) by the large scale structure of the magnetic field, for example a helix, where linearly polarized radiation from the back of the jet presents (depending on the pitch angle) as Stokes U at the front of the jet.

The first paper that reported the detection of circular polarization at milliarcsecond resolution [98] suggested that this was evidence for a pair dominated jet. The essence of the argument was that the observed levels of linear and circular polarization seemed to require (after extensive Monte Carlo simulations) that the low energy cutoff in the electron energy distribution $\gamma_{\min } \leq 20$. Extrapolating the radiating particle spectrum to such low energies meant that if the positive particles were protons, then the jet would carry one or two orders of magnitude more kinetic energy than appeared to be dissipated in the extended radio source.

Beckert \& Falke [99] using a magnetic field model with a strong helical component in an electron-proton jet, also found that $\gamma_{\min } \approx 20$ but it did not need to be lower. Ruszkowski \& Begelman [100] used a different magnetic field model with small scale turbulence and an admixture of cold electrons, and could match the observations with an electron-proton jet with $\gamma_{\min } \approx 30$. All these results are not so much in disagreement as show that there is a large parameter space to be explored. They all demonstrate that these observations are sensitive to the otherwise unobservable low energy end of the relativistic electron distribution.

When a spectrum in each Stokes parameter is measured, then the models are much more severely constrained. This is the case in [82] which reported observations of 3C279 at six frequencies between 8.01 and 24.35 $\mathrm{GHz}$ The spectra in total intensity, linear and circular polarization (and rotation measure) for each component were modeled by radiative transfer simulations to find a selfconsistent description of the magnetic field structure and particle content of the jet. The reader is referred to that paper for details, but one particularly interesting result is that the net magnetic flux of the poloidal component was determined to be in the range $2 \times 10^{34}$ to $1 \times 10^{35} \mathrm{G} \mathrm{cm}^{2}$. Presumably this is also the magnetic flux at the central engine. 


\section{Summary}

There are many aspects of the polarization properties of AGN on parsec scales that space does not permit to mention here. These include the technique of Faraday synthesis, and the prospect of the Event Horizon Telescope imaging polarized emission in the strong gravitational field of the supermassive black holes at the centers of the Milky Way and M87.

The results discussed here include the following conclusions:

(1) We suggest that while many of the observations of transverse rotation measure gradients may be correct, they have in general been observed with neither enough resolution nor sensitivity to be incontrovertible. Fortunately, this could be remedied using the new broad-band receivers on the VLBA.

(2) We also argue that while a transverse rotation measure gradient is a strong indicator of a toroidal component of magnetic field, it does not necessarily imply a helical magnetic field. In an expanding jet a helix is rapidly wound up to a pitch angle $\approx 90^{\circ}$. In jets where the dominant field appears to be parallel to the jet, it most likely consists of sheared loops or compressed random fields that are not vector ordered.

(3) With care, reliable measurements of circular polarization can be made. If they are combined with measurements of the other three Stokes parameters at multiple frequencies, then detailed modeling can strongly constrain the magnetic field structure and particle content in the source in ways that are not possible without the circular polarization measurements.

And finally: (4) only one epoch of MOJAVE linear polarization images has been published, as has only one epoch of circular polarization images or rotation measure images. The time domain has barely been scratched, (though other observers have made considerable headway with a small number of sources). Most MOJAVE sources have more than 20 observations over more than 10 years. This is in no way a criticism, but rather an anticipation of the advances to our understanding of jet physics that must lie in those data.

\section{Acknowledgements}

I would like to thank Avery Broderick, Denise Gabuzda, Dan Homan, Talvikki Hovatta, Seguei Komissarov, Matt Lister and Al Marscher for helpful comments, clarifications and conversations, and José-Luis Gómez and the organizers for this excellent meeting.

\section{References}

[1] Zensus, J. A., Ann. Rev. Astron. \& Astrophys. 35, 607 (1997)

[2] Romney, J. \& Reid, M., Future Directions in High Resolution Astronomy: ASP Conf. 340 (2005)

[3] Lister, M. L. \& Homan, D. C., AJ 130, 1389 (2005)

[4] Jorstad et al., ApJS 134, 181 (2001)
[5] O’Sullivan,S., Gabuzda, D. C. \& Gurvits, L. I., MNRAS 415, 3049 (2011)

[6] Roberts, D. H., Wardle, J. F. C. \& Brown, L. F., ApJ 427 (1994)

[7] Homan, D. C. \& Lister, M. L., AJ 131, 1262 (2006)

[8] Hovatta, T., et al. AJ 144, 105 (2012)

[9] Mahmud, M. et al., MNRAS 431, 695 (2013)

[10] Cawthorne, T. V. et al., ApJ 416, 519 (1993)

[11] Pollack, L. K., Taylor, G. B. \& Zavala, R. T., ApJ 589, 733 (2003)

[12] Helmboldt, J. et al., ApJ 658, 203 (2007)

[13] Lister, M. L., ApJ 562, 208 (2001)

[14] Pearson, T. J. \& Readhead, A. C. S., ApJJ 328, 114 (1988)

[15] Avni, Y. et al., ApJ 238, 800 (1980)

[16] Wardle, J., et al., ApJ 437, 122 (1994)

[17] Laing, R. A., MNRAS 193, 439 (1980)

[18] Hughes, P. A, Aller, H. D. \& Aller, M. F., ApJ 341, 68 (1989)

[19] Bridle, A. H. \& Perley, R. A, Ann. Rev. Astron. \& Astrophys 22, 319 (1984)

[20] Begelman, M. C., Blandford, R. D. \& Rees, M. J., Rev. Mod. Phys. 56, 255 (1984)

[21] Jorstad et al., AJ 134, 799 (2007)

[22] Barthel, P. D., ApJ 336, 606 (1989)

[23] Kharb, P., Shastri, P. \& Gabuzda, D. C., ApJ 632, 69 (2005)

[24] Middelberg, E., PhD thesis, University of Bonn (2004)

[25] Zavala, R. T. \& Taylor, G. B., ApJ 549, 146 (2003)

[26] Blandford, R. D. \& Königl, A., ApJ 232, 34 (1979)

[27] Cawthorne, T. V. \& Cobb, W., ApJ 350, 536 (1990)

[28] Cawthorne, T. V., MNRAS 367, 851 (2006)

[29] Bicknell, G. V. \& Begelman, M. C., ApJ 467, 597 (1996)

[30] Agudo, I. et al., ApJ 549, 183 (2001)

[31] Hughes, P. A., ApJ 621, 635 (2005)

[32] Hughes, P. A, Aller, H. D. \& Aller, M. F., ApJ 735, 81 (2011)

[33] Roberts, D. H. \& Wardle, J. F. C. ApJ 759, L35 (2012)

[34] Gabuzda, D. C., et al., MNRAS 369, 1596 (2006)

[35] D'Arcangelo, F. et al., ApJ 659, 107 (2007)

[36] Marscher, A. P., et al., Nature 452, 966 (2008)

[37] Marscher, A. P. et al., ApJ 710, 126 (2010)

[38] Orienti, M. et al., MNRAS 428, 2418 (2013)

[39] Larionov, V. M. et al., ApJ 768, 40 (2013)

[40] Vlahakis, N., in Blazar Variability Workshop II: Entering the GLAST Era, ed. H. R. Miller et al., ASP Conf. 350, 169 (2006)

[41] Vlahakis, N. \& Königl, A, ApJ 605, 656 (2004)

[42] Komissarov, S. S., et al., MNRAS 380, 51 (2007)

[43] Taylor, G. B., ApJ 506, 637 (1998)

[44] Taylor, G. B., ApJ 533, 95 (2000)

[45] Zavala, R. T. \& Taylor, G. B., ApJ 550, L147 (2001)

[46] Homan, D. C., ApJL 747, L24 (2012) 
[47] O’Sullivan, S. P. \& Gabuzda, D. C., MNRAS 393, 429 (2009)

[48] Algaba, J., Gabuzda, D. \& Smith, P., MNRAS 411, 85 (2011)

[49] Algaba, J., Gabuzda, D. \& Smith, P., MNRAS 420, 542 (2012)

[50] Königl, A., ApJ 243, 700 (1981)

[51] Kovalev, Y.Y., et al., A\&A 483, 759 (2008)

[52] Pushkarev, A. B. et al. A\&Ap 545, 113 (2012)

[53] Wardle, J. F. C., Nature 269, 563 (1977)

[54] Jones, T. W. \& O’Dell, S. L., ApJ 214, 522 (1977)

[55] Gómez, J.-L. et al., ApJ 681, L69 (2008)

[56] Asada, K. et al., PASJ 54, L39 (2002)

[57] Zavala, R. T. \& Taylor, G. B., ApJ 626, L73 (2005)

[58] Attridge, J. M., Wardle, J. F. C. \& Homan, D. C., ApJ 633, 85 (2005)

[59] Blandford, R., in Astrophysical Jets, Astrophysics and Space Science Library (Cambridge: Cambridge Univ. Press), 103, 15(1993)

[60] Contopoulos, I., et al., ApJ 702, L148 (2009)

[61] Taylor, G. B., \& Zavala, R., ApJ 722, L183 (2010)

[62] Broderick,, A. E. \& McKinney, J. C., ApJ 725, 750 (2010)

[63] Murphy, E. \& Gabuzda, D., Journal of Physics: Conference Series, 355, Issue 1 (2012)

[64] Murphy, E., et al., arXiv:1309.1718 (2013)

[65] Hovatta, T., et al. AJ 145, 172 (2013)

[66] Chen, T., Ph.D. thesis, Brandeis Univ. (2005)

[67] Asada, K. et al., ApJ 675, 79 (2008)

[68] Gómez, J.-L. et al., ApJ 733, 11 (2011)

[69] Mahmud, M., Gabuzda, D. \& Bezrukovs, V., MNRAS 400, 2 (2009)

[70] Lynden-Bell, D., MNRAS 279, 389 (1996)

[71] Pushkarev, A., et al., A\&Ap Letters 507, L33 (2009)

[72] Laing, R. A., ApJ 248, 87 (1981)

[73] Clausen-Brown, E., Lyutikov, M. \& Kharb, P., MNRAS 415, 2081 (2011)

[74] Cohen, M. H., in BL Lac Objects, Lecture Notes in Physics (Berlin: Springer-Verlag) 334, 13 (1989)
[75] Broderick, A. E. \& Loeb A., ApJ 703, L104 (2009)

[76] Homan, D. C. et al., ApJ 706, 1253 (2009b)

[77] Lister, M. L. et al. arXiv:1308.2713 (2013)

[78] Savolainen, T., et al., A\&Ap 446, 71 (2006)

[79] Begelman, M. C., in Jets in Extragalactic Radio Sources, Lecture Notes in Physics (Berlin:SpringerVerlag) 421, 145 (1991)

[80] Laing, R. A. \& Bridle, A. H., MNRAS 336, 1161 (2002)

[81] Laing, R. A., Canvin, J. R. \& Bridle, A. H., Astron. Nacht. 327, 523 (2006)

[82] Homan, D. C. et al., ApJ 696, 328 (2009a)

[83] Mizrahi, J, Senior honors thesis, Brandeis University (2007)

[84] Homan, D. C. \& Wardle, J. F. C., AJ 118, 1942 (1999)

[85] Homan, D. C., Attridge, J. A. \& Wardle, J. F. C., ApJ 556, 113 (2001)

[86] Komesaroff, M. M., et al. MNRAS 208. 409 (1984)

[87] Rayner, D. P., Norris, R. P., \& Sault, R. J., MNRAS 319, 484 (2000)

[88] Macquart, J.-P., et al., ApJ 538, 623 (2000)

[89] Macquart, J.-P. \& Melrose, D. B., ApJ 545, 798 (2000)

[90] Gabuzda, D. C., et al., MNRAS 384, 1003 (2008)

[91] Vitrishchak et al., MNRAS 391, 124 (2008)

[92] Legg, M. P. C. \& Westfold, K. C., ApJ 154, 499 (1968)

[93] Wardle, J. F. C.\& Homan, D. C. Ap\&SS 288, 143 (2003)

[94] Enßlin, T. A., A\&A 401, 499 (2003)

[95] Aller, H. D., Aller, M. F. \& Plotkin, R. M., Ap\&SS 288, 17 (2003)

[96] Sikora, M. \& Madejski, G., ApJ, 534, 534 (2000)

[97] Jones, T. W. ApJ 332, 678 (1988)

[98] Wardle, et al., Nature 395, 457 (1998)

[99] Beckert, T. \& Falke, H., A\&A 388, 1106 (2002)

[100] Ruszkowski, M. \& Begelman, M. C. ApJ 573, 485 (2002) 